Dental Cadmos, vol. 85, núm. 3, 2017, pp. 136-143.

\title{
Eventi avversi in ortodonzia: revisione della letteratura.
}

\author{
Vaquero Niño, Paula, Perea Pérez, Bernardo, Labajo \\ González, Elena y Santiago Sáez, Andrés.
}

Cita:

Vaquero Niño, Paula, Perea Pérez, Bernardo, Labajo González, Elena y Santiago Sáez, Andrés (2017). Eventi avversi in ortodonzia: revisione della letteratura. Dental Cadmos, 85 (3), 136-143.

Dirección estable: https://www.aacademica.org/elenalabajogonzalez/34 ARK: https://n2t.net/ark:/13683/pcQr/xDr

https://creativecommons.org/licenses/by-nc-nd/4.0/deed.es.

Acta Académica es un proyecto académico sin fines de lucro enmarcado en la iniciativa de acceso abierto. Acta Académica fue creado para facilitar a investigadores de todo el mundo el compartir su producción académica. Para crear un perfil gratuitamente o acceder a otros trabajos visite: https://www.aacademica.org. 


\title{
Eventi avversi in ortodonzia: revisione della letteratura
}

\section{Adverse events in orthodontic practice: a review of the literature}

\author{
P. Vaquero-Niñoa, B. Perea-Pérez, E. Labajo-González $z^{\mathrm{b},}$, A. Santiago-Sáez \\ a Università Complutense di Madrid, Dipartimento di Stomatologia Il; Osservatorio Spagnolo per la Sicurezza Dentale del Paziente (OESPO) \\ ' Università Complutense di Madrid, Scuola di Medicina Legale e Forense di Madrid; Osservatorio Spagnolo per la Sicurezza Dentale del Paziente \\ (OESPO)
}

Ricevuto il 24 marzo 2016

\begin{abstract}
RIASSUNTO
Obiettivi. L'obiettivo della presente revisione della letteratura è quello di determinare gli eventi awersi derivanti dalla pratica ortodontica, anche se in letteratura non è frequente riscontrare la descrizione di tali eventi. Materiali e metodi. La ricerca degli articoli è stata realizzata nel database PubMed utilizzando le parole chiave "adverse event", "adverse events" e "orthodontics" separate dall'operatore booleano "AND". Verificando i criteri di inclusione e di validità delle variabili in base ai quali sono stati cercati i dati, i riferimenti bibliografici sono stati gestiti accertando nel riassunto la specificità del tipo di studio, del campione, dei risultati, della discussione e delle conclusioni. Risultati. La ricerca iniziale ha prodotto 68 citazioni, di cui 49 sono state eliminate perché non soddisfacevano i criteri di inclusione; i restanti 19 studi sono stati scelti per l'analisi qualitativa. Poiché questi ultimi sono stati condotti utilizzando metodologie differenti, non è stato possibile realizzare un'analisi quantitativa. I principali eventi awersi presi in esame sono stati: riassorbimento radicolare, danno pulpare, danno parodontale, inalazione o ingestione di apparecchiature ortodontiche, ipersensibilità al nichel e ad altri materiali ortodontici, ipersensibilità al paracetamolo, limitazioni al movimento dentale e disfunzione cranio-mandibolare. Discussione e conclusioni. Dalla rassegna emerge límportanza della documentazione medica e dentale, della documentazione diagnostica completa, del consenso informato scritto e di un corretto protocollo di trattamento. La maggior parte degli eventi awersi associati all'ortodonzia sono di bassa o moderata severità. In molti casi gli eventi awersi possono essere evitati.
\end{abstract}

Parole chiave: Ortodonzia | Sicurezza del paziente | Eventi avversi | Riassorbimento radicolare | Ipersensibilità

\section{ABSTRACT}

Objectives. Adverse events in orthodontic practice are infrequently described in the literature. The objective of this review is to determine and analyse such adverse events. Materials and methods. The search for articles was performed using the PubMed 
database. The key words were "adverse event," "adverse events" and "orthodontics", separated by the Boolean operator "AND". The criteria of inclusion and validity of variables, by which the data were sought, permitted to select the references by verifying in the Summary the specification of study type, sample, results, discussion and conclusions. Results. The initial search produced 68 records, 49 of which were eliminated because they did not meet the criteria for inclusion, whereas 19 were included in the qualitative analysis. Because these studies used different methodologies, it was not possible to complete a quantitative analysis. The main adverse events included were root resorption, pulp damage, periodontal damage, inhalation or ingestion of orthodontic products and apparatuses, hypersensitivity to nickel and to other orthodontic materials, hypersensitivity to paracetamol, restricted jaw movement and craniomandibular dysfunction. Discussion and conclusions. The study highlights the importance of keeping proper medical and dental records, complete diagnostic records, informed consent forms and proper treatment protocols. Most adverse events associated with orthodontic practice are of low to moderate severity and are avoidable in most cases.

Key words: Orthodontics | Patient safety | Adverse events | Root resorption | Hypersensitivity

\section{Introduzione}

Nei primi anni Novanta un lavoro di Leape et al. [1] evidenziò come i due terzi degli eventi awersi che erano stati segnalati nella pratica medica fossero evitabili. Anche se i danni che i trattamenti medici avrebbero potuto causare erano noti da sempre, solo da quel momento si manifestò la consapevolezza della reale importanza del problema e si iniziò a quantificarlo. Un altro studio fondamentale, in termini di crescente consapevolezza del problema, fu quello portato a termine nel 1999 dall'Institute of Medicine of the National Academics [2], nel quale si sottolineava che negli Stati Uniti, a seguito delle stesse cure sanitarie, si era verificato un numero di decessi evitabili compre. so tra 44.000 e 98.000 casi.

L'Organizzazione Mondiale della Sanità definisce la sicurezza del paziente come la riduzione a un minimo accettabile del rischio di danni inutili connessi con l'assistenza sanitaria, il che chiama in causa il grado delle conoscenze collettive nel momento in cui si esegue la procedura, le risorse disponibili e l'ambiente in cui si eroga la prestazione sanitaria, il tutto confrontato con il rischio del mancato trattamento o del ricorso a qualche altro tipo di trattamento [3]. La sicurezza del paziente riveste grande importanza nella pratica odontoiatrica alla luce sia della rapida innovazione nei metodi e nei materiali, della prescrizione di nuovi farmaci, dellintroduzione di nuovi approcci terapeutici, che stanno diventando più invasivi allo scopo di trovare soluzioni per patologie gravi, sia degli interventi di riabilitazione orale sempre più complessi [4].

In odontoiatria il tema della sicurezza del paziente sta assumendo grande importanza e producendo una crescente ripercus-
Sione tra i professionisti. L'obiettivo fondamentale è quello di scongiurare al massimo grado possibile gli eventi avversi evitabili (incidenti, errori o complicazioni) associati alle cure odontoiatriche [5]. In ortodonzia, il riscontro di eventi awersi riportati in letteratura non è frequente. La maggior parte di questi riguarda il riassorbimento radicolare indotto dal trattamento [6-8], la necrosi pulpare dovuta a forze eccessive di attivazione esercitate dalle apparecchiature, il danno parodontale [9], l'inalazione o ingestione di apparecchi ortodontici [8], lipersensibilità al nichel e ad altri materiali ortodontici [10-12].

A causa delle implicazioni medico-legali che tutto ciò comporta, sono state pubblicate alcune raccomandazioni sui metodi per evitare o limitare la loro comparsa [13-16]

In letteratura esistono alcuni studi che riportano eventi awersi, tuttavia manca una revisione sistematica che identifichi quelli con massima prevalenza e che incidono sulla sicurezza del paziente ortodontico. Scopo della presente revisione della letteratura è pertanto determinare gli eventi awersi derivanti dalla pratica ortodontica ed evidenziare le variabili cliniche correlate alla loro evenienza, oltre a promuovere futuri studi di metanalisi.

\section{Materiali e metodi}

Questo studio è stato condotto nell'ambito del progetto di ricerca Progettazione e sviluppo di un osservatorio per la sicurezza dentale del paziente in ambito nazionale, un progetto della Scuola UCM di Medicina Legale e del Consiglio Generale degli Odontoiatri e Stomatologi di Spagna.

II sistema PICO per la formulazione dei quesiti clinici si basa sulla trasformazione delle esigenze conoscitive del clinico su un tema 
specifico utilizzando quattro componenti: Paziente, Intervento, Confronto e Outcome. Gli studi che hanno valutato le esigenze di informazione nella pratica clinica concordano nel ritenere che fino all' $80 \%$ delle richieste abbia a che fare con il processo diagnostico e la scelta del trattamento. II format utilizzato per ideare il quesito PICO è riportato in tabella I. I criteri di ammissione degli studi nella presente revisione si sono basati sul rispetto dei quattro item del quesito PICO. Non vi sono state restrizioni in termini di data, autore o stato di pubblicazione.

La ricerca degli articoli pubblicati da riviste scientifiche è stata effettuata utilizzando la banca dati PubMed nel periodo di tempo compreso tra il gennaio 2010 e il gennaio 2016.

La ricerca in PubMed è stata svolta utilizzando contemporaneamente le parole chiave "adverse event", "adverse events" e "orthodontics" separate dall'operatore booleano "AND". Da questa ricerca iniziale sono emersi 75 risultati (con 7 citazioni doppie eliminate). In tale ricerca non era stata specificata alcuna limitazione.

I criteri di inclusione degli studi sono stati i seguenti:

- articoli comprendenti eventi awersi in pazienti ortodontici (di qualsiasi età e genere e con qualsiasi tipo di malocclusione):

- qualsiasi pubblicazione;

- qualsiasi tipo di studio;

- lingua inglese (rilevata dalla lingua utilizzata nel database PubMed).

Il procedimento per la raccolta dei dati dalle pubblicazioni è stato effettuato in maniera separata. Le variabili utilizzate per la ricerca dei dati sono riportate in tabella II.

La valutazione del rischio di bias nei singoli studi è stata condotta a livello degli stessi studi e dei loro risultati, senza alcun influsso da parte dei risultati pubblicati in precedenza, e sono state analizzate le potenziali fonti di finanziamento con conflitti di interesse, quando ritenuto opportuno.

Una volta che sono stati verificati i criteri per linclusione e la validità delle variabili con $i$ quali erano stati ricercati $i$ dati, questi ultimi sono stati trattati secondo verifica del tipo di studio, campione, risultati, discussione e conclusioni corrispondenti alle voci del riassunto. Se tutte le premesse erano soddisfatte, i risultati venivano raggruppati.

\section{Risultati}

La ricerca elettronica iniziale ha riportato 68 citazioni singole. Non erano presenti articoli o riferimenti doppi. Le 68 citazioni bibliografiche sono state selezionate leggendo il titolo e il rias-

\begin{tabular}{l|l}
\hline Tab.I & Format PICO \\
\hline Pazienti & $\begin{array}{l}\text { Pazienti senza trattamenti ortodontici pregressi o altre lesioni } \\
\text { associate (riassorbimento radicolare, necrosi pulpare, danno } \\
\text { parodontale) di qualsiasi età, genere e con qualsiasi tipo } \\
\text { di malocclusione }\end{array}$ \\
\hline Intervento & $\begin{array}{l}\text { Trattamento ortodontico, indipendentemente dagli } \\
\text { apparecchi usati }\end{array}$ \\
\hline Confronto & $\begin{array}{l}\text { Pazienti che hanno ricevuto un trattamento ortodontico } \\
\text { Outcome }\end{array}$ \\
\hline
\end{tabular}

\begin{tabular}{l|l|}
\hline Tab.II & Variabili utilizzate per la ricerca dei dati \\
\hline Variabile & Definizione \\
\hline $\begin{array}{l}\text { Metodo } \\
\text { di randomizzazione }\end{array}$ & $\begin{array}{l}\text { Qualsiasi sequenza casuale che non lasci prevedere } \\
\text { il numero del caso o la data di nascita } \\
\text { Soggetti che potrebbero trarre beneficio da un } \\
\text { trattamento ortodontico, senza limitazioni riguardo } \\
\text { al tipo o al grado di malocclusione, alleta o al genere }\end{array}$ \\
\hline $\begin{array}{l}\text { Irattamento ortodontico, indipendentemente } \\
\text { dagli apparecchi usati }\end{array}$ \\
\hline $\begin{array}{l}\text { Pazienti trattati in ortodonzia } \\
\text { Riscontro di eventi awersi durante la pratica } \\
\text { Ortodontica }\end{array}$ \\
\hline Outcome
\end{tabular}

sunto; 49 di queste sono state eliminate perché non soddisfacevano i criteri di inclusione [17-19]. Al termine, il numero totale di studi inclusi nell'analisi qualitativa della revisione è stato di 19 lavori: 1 metanalisi, 2 revisioni sistematiche, 1 trial clinico randomizzato controllato, 2 studi clinici di confronto (uno dei quali parte di uno studio multicentrico), 7 articoli di revisione, 4 casi clinici (in cui ogni articolo descriveva un solo caso) e 2 casi forensi [8-12,20-33] (fig. 1). Poiché in questi studi sono state utilizzate metodologie diverse, non è stato possibile realizzare l'analisi quantitativa. Ė stato stabilito che il rischio di errori sistematici (bias) era basso per gli studi inclusi nella revisione.

II riscontro di eventi awersi nella pratica ortodontica presenti in letteratura è sintetizzato in tabella III.

\section{Discussione}

Innanzitutto, l'attenzione degli autori si è soffermata sul numero relativamente basso di pubblicazioni riguardanti gli eventi avversi che occorrono durante i trattamenti ortodontici.

\subsection{Eventi avversi associati alla pratica ortodontica riportati in letteratura}

\section{$>$ Prevalenza e grado di riassorbimento radicolare}

Per quanto riguarda il riassorbimento radicolare, una delle difficoltà nel determinare l'agente causale è la distinzione tra il con- 


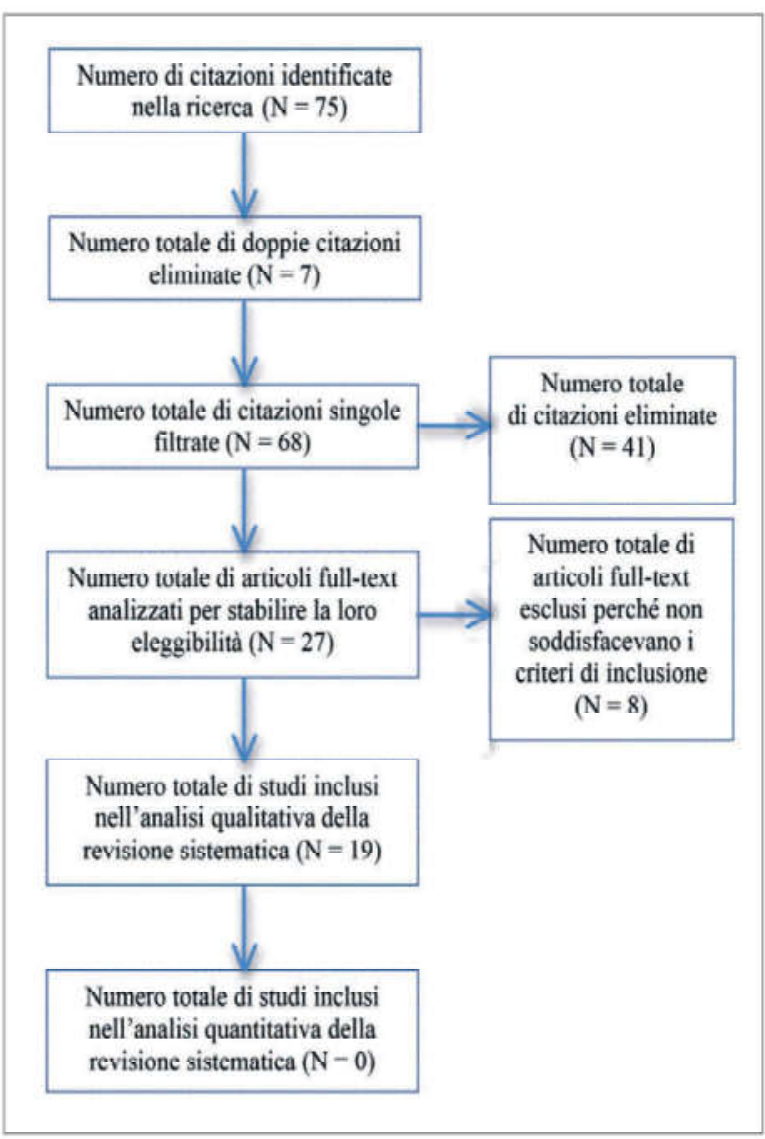

Fig. 1 Diagramma di flusso

delle differenti fasi della revisione della letteratura

tributo dei fattori genetici e quello dei fattori ambientali, quali il trattamento e le cure. Al fine di monitorare il riassorbimento radicolare occorre eseguire controlli radiografici 6 mesi dopo l'inizio del trattamento. In pazienti con fattori di rischio, l'esame radiografico va fatto dopo 3 mesi. Inoltre, in base all'associazione dellinterleuchina-1B con lo sviluppo di queste lesioni, tale citochina potrebbe essere un marcatore genetico per eseguire un test di suscettibilità al disturbo [24,25].

II riassorbimento radicolare è un fenomeno comune associato al trattamento ortodontico e può iniziare nelle prime fasi del trattamento [24,25]. La sua prevalenza varia notevolmente a seconda degli studi $[24,25]$. Sono stati riportati valori medi che vanno da 0,5 a $3 \mathrm{~mm}$ di riduzione della radice. La frequenza del riassorbimento severo oscilla dal 5\% al 18\% [24]. La prevalenza e il grado di riassorbimento dipendono da: dispositivi utilizzati, torque radicolare, tipo di forza, tipo di movimento, entità della forza e tempo di trattamento $[24,25]$. II consumo di alcol e l'assunzione di corticosteroidi durante il trattamento attivo aumentano il riassorbimento radicolare [8], mentre la somministrazione di tiroxina diminuisce il rischio di sviluppare queste lesioni $[8,24]$. Inoltre, in pochi casi la riduzione radicolare è grave durante la terapia con apparecchi fissi [6].

La diagnosi di questo evento awerso si effettua prevalentemente mediante radiografia panoramica e periapicale e teleradiografia laterale [25].

Nel 1984 Brady et al. [23] hanno riportato un caso di riassorbimento radicolare interno in un incisivo superiore centrale in un paziente con parodontite cronica e perdita di osso alveolare. Nel 1990 Machen [29] ha pubblicato un caso di negligenza professionale da parte di un ortodontista che aveva eseguito un trattamento con perdita di supporto parodontale e riassorbimento radicolare causati dall'applicazione di forze di entità eccessiva durante il trattamento attivo e fallimento del trattamento al follow-up.

Nel 1991 Mostafa et al. [27] hanno pubblicato uno studio clinico randomizzato volto a indagare le reazioni pulpari alle forze di estrusione ortodontiche nei primi premolari mascellari rimossi, concludendo che la polpa reagisce andando incontro a degenerazione degli odontoblasti, vasodilatazione, vacuolizzazione ed edema tessutale.

\section{$>$ Problemi parodontali}

Talvolta, dopo un movimento ortodontico in direzione labiale, si produce una deiscenza ossea con perdita della mucosa cheratinizzata sulla superficie vestibolare dei denti $[9,26]$. In altri casi vi è una perdita totale della mucosa cheratinizzata in seguito a un trattamento ortodontico. Ciò è stato dimostrato da Castro et al. [26] a livello dei canini inferiori. Vi è anche un rapporto tra la comparsa di recessioni gengivali e l'uso di dispositivi ortodontici. Questo tipo di lesione può verificarsi come pseudorecessione o recessione effettiva. È necessaria una valutazione dei fattori che creano una predisposizione a entrambe le condizioni al fine di stabilire se la chirurgia sia indicata $[9,26]$. Allo stesso modo, si è riscontrata una riduzione significativa di tessuto cheratinizzato in bambini sottoposti a trattamento con apparecchi funzionali [9].

Sempre in tema di problemi parodontali, de Castro et al. [26] hanno riportato il caso di una cisti con inclusione epiteliale dopo innesto gengivale libero eseguito con l'obiettivo di bloccarne la recessione per poter proseguire un trattamento orto- 
Tab. III Riscontro di eventi avversi durante la pratica ortodontica riportati in letteratura (dalla tabella sono state escluse una metanalisi e due revisioni sistematiche, a causa della duplicazione dei risultati, e anche i due casi forensi)

\begin{tabular}{|c|c|c|c|c|c|c|c|c|c|}
\hline $\begin{array}{l}\text { Autore, } \\
\text { anno }\end{array}$ & Paese & Popolazione & $\begin{array}{l}\mathrm{N} \text {. } \\
\text { pazienti }\end{array}$ & $\begin{array}{l}\text { Età } \\
\text { dei pazienti } \\
\text { (anni) }\end{array}$ & Genere & $\begin{array}{l}\text { Malocclu- } \\
\text { sione }\end{array}$ & $\begin{array}{l}\text { Apparecchi } \\
\text { ortodontici }\end{array}$ & Evento averso & Trattamento medico \\
\hline $\begin{array}{l}\text { Bilder et al., } \\
2011[10]\end{array}$ & - & - & 21 & $\begin{array}{l}\text { Maschi: } 12,25 \\
\text { (media) } \\
\text { Femmine: } 13,83 \\
\text { (media) }\end{array}$ & $\begin{array}{l}\text { M9 } \\
\text { F12 }\end{array}$ & - & $\begin{array}{l}\text { Apparecchi fissi } \\
\text { Apparecchi mobili }\end{array}$ & $\begin{array}{l}\text { Inalazione } \\
\text { Ingestione di strumenti } \\
\text { ortodontici (chiavi } \\
\text { per attivazione di apparecchi } \\
\text { mobili) }\end{array}$ & $\begin{array}{l}\text { Trattamento medico nel } 79 \% \\
\text { dei casi } \\
\text { Esame radiografico } \\
\text { Esofagoscopia, endoscopia, } \\
\text { broncuscopia }\end{array}$ \\
\hline $\begin{array}{l}\text { Borromeo et al., } \\
2011 \text { [31] }\end{array}$ & - & - & 4 & - & - & - & Apparecchi fissi & $\begin{array}{l}\text { Limitazione del movimento } \\
\text { dentale }\end{array}$ & - \\
\hline $\begin{array}{l}\text { Brady et al., } \\
1984 \text { [23] }\end{array}$ & Inghilterra & Caucasica & 1 & 34 & F & $\begin{array}{l}\text { Classe ll, } \\
\text { Divisione } 1\end{array}$ & Apparecchi mobili & $\begin{array}{l}\text { Riassorbimento radicolare } \\
\text { interno dellincisivo centrale } \\
\text { superiore }\end{array}$ & $\begin{array}{l}\text { Ferulizzazione e chirurgia } \\
\text { parodontale } \\
\text { Trattamento endodontico }\end{array}$ \\
\hline $\begin{array}{l}\text { Counts etal. } \\
2002[11]\end{array}$ & USA & Caucasica & 1 & 11 & F & $\begin{array}{l}\text { Classe III } \\
\text { emorso } \\
\text { aperto }\end{array}$ & $\begin{array}{l}\text { Barra } \\
\text { transpalatale }\end{array}$ & Allergia al nichel & $\begin{array}{l}\text { Rimozione degli apparecchi } \\
\text { Gengivectomia } \\
\text { Test per rallergia al nichel }\end{array}$ \\
\hline $\begin{array}{l}\text { De Castro et al, } \\
2007[26]\end{array}$ & Brasile & Sudamericana & 1 & 22 & F & - & $\begin{array}{l}\text { Apparecchi fissi } \\
\text { multibracket }\end{array}$ & $\begin{array}{l}\text { Danno parodontale } \\
\text { (perdita totale della mucosa } \\
\text { cheratinizzata) }\end{array}$ & Innesto libero di gengiva \\
\hline $\begin{array}{l}\text { Fricton et al., } \\
2012[21]\end{array}$ & - & - & - & - & - & Classe II & $\begin{array}{l}\text { Apparecchi } \\
\text { funzionali } \\
\text { ortopedici } \\
\text { intraorali }\end{array}$ & $\begin{array}{l}\text { Sviluppo di ulcere mucose, } \\
\text { dolore dentale, difficoltà } \\
\text { a parlare, carie, alitosi, } \\
\text { mobilita dentale }\end{array}$ & - \\
\hline $\begin{array}{l}\text { Machen, } \\
1989[30]\end{array}$ & USA & Caucasica & 1 & 26 & F & Classe II & $\begin{array}{l}\text { Apparecchi } \\
\text { fissi }\end{array}$ & $\begin{array}{l}\text { Danno parodontale (perdita } \\
\text { ossea) e rassorbimento } \\
\text { radicolare }\end{array}$ & - \\
\hline $\begin{array}{l}\text { Machen, } \\
1990 \text { [29] }\end{array}$ & USA & Caucasica & 1 & 40 & F & $\begin{array}{l}\text { Classe II, } \\
\text { Divisione } 2\end{array}$ & $\begin{array}{l}\text { Apparecchi } \\
\text { fissi }\end{array}$ & $\begin{array}{l}\text { Dolore miofasciale. } \\
\text { Disfunzione cranio-mandibolare }\end{array}$ & - \\
\hline $\begin{array}{l}\text { McAlinden et al, } \\
2005[22]\end{array}$ & Inghilterra & Caucasica & 1 & 12 & M & - & $\begin{array}{l}\text { Apparecchi } \\
\text { fissi }\end{array}$ & $\begin{array}{l}\text { Ipersensibilità al paracetamolo } \\
\text { Eruzione cutanea sullintero } \\
\text { corpo e prurito }\end{array}$ & $\begin{array}{l}\text { Somministrazione } \\
\text { di antistaminici }\end{array}$ \\
\hline $\begin{array}{l}\text { Moriarty. } \\
1996[9]\end{array}$ & USA & Caucasica & 1 & 14 & - & - & $\begin{array}{l}\text { Apparecchi } \\
\text { fissi }\end{array}$ & $\begin{array}{l}\text { Recessione e lacerazione } \\
\text { gengivale }\end{array}$ & $\begin{array}{l}\text { Innesto autologn con margine } \\
\text { cheratinizzato intatto }\end{array}$ \\
\hline $\begin{array}{l}\text { Mostafa et al., } \\
1991 \text { [27] }\end{array}$ & Egitro & Africana & 18 & 18 (media) & - & - & $\begin{array}{l}\text { Apparecchi } \\
\text { fissi }\end{array}$ & $\begin{array}{l}\text { Danno pulpare: degenerazione } \\
\text { degli osteoblasti, } \\
\text { vasodilatazione, edema }\end{array}$ & - \\
\hline $\begin{array}{l}\text { Thavarajah et al, } \\
\text { 2015 [28] }\end{array}$ & India & - & 175 & - & - & - & $\begin{array}{l}\text { Allineatorí } \\
\text { sequenziali }\end{array}$ & $\begin{array}{l}\text { Reazione allergica } \\
\text { Reazione anafilattica } \\
\text { Angioedema } \\
\text { Indolenzimento } \\
\text { Infiammazione } \\
\text { Orticaria } \\
\text { Nausea } \\
\text { Disturbi gastrointestinali } \\
\text { Disturbi neuromusculari } \\
\text { Tosse } \\
\text { Cefalea persistente } \\
\text { Febbre } \\
\text { Disturbi correlati al cuore }\end{array}$ & - \\
\hline $\begin{array}{l}\text { Thiruvenkatachan } \\
\text { et al, } 2010[20]\end{array}$ & Inghilterra & Caucasica & 31 & 12 (media) & - & $\begin{array}{l}\text { Classell, } \\
\text { Divisione } 1\end{array}$ & $\begin{array}{l}\text { Applicazioni } \\
\text { dynamax } \\
\text { (26 effetti awersi) } \\
\text { Applicazioni } \\
\text { twin-block (5) }\end{array}$ & $\begin{array}{l}\text { Rottura degli apparecchi } \\
\text { Trauma alla mucosa linguale } \\
\text { e alla lingua }\end{array}$ & - \\
\hline $\begin{array}{l}\text { Toddet al, } \\
1989 \text { [12] }\end{array}$ & Iflanda & Caucasica & $\pi$ & - & $\begin{array}{l}M 2 \\
F 75\end{array}$ & - & $\begin{array}{l}\text { Apparecchi fissi } \\
\text { multibracket }\end{array}$ & Allergia al nichel & - \\
\hline
\end{tabular}

140 | DeNTALCADMOS | 3/2017 
dontico. La comparsa di lesioni cistiche secondarie alla tecnica di innesto gengivale non è un fenomeno molto frequente. Sarebbe interessante approfondire il fatto che la maggior parte di esse appare a volte in pazienti ortodontici nella zona del primo premolare-canino-incisivo inferiore. L'indicazione all'intervento chirurgico per le recessioni gengivali, sfortunatamente, non può essere stabilita in modo obiettivo in quanto molti parametri non sono valutati mediante misurazioni oggettive. I criteri oggettivi comprendono l'anatomia della zona, lo spessore delle gengive cheratinizzate, la dimensione vestibolo-linguale del tessuto molle e alveolare, la posizione e l'angolazione del dente in questione [9].

\section{> Inalazione e ingestione di apparecchi ortodontici o di loro elementi}

L'ingestione di oggetti non comporta di norma gravi problemi medici, mentre l'inalazione determina un reale pericolo in relazione al rischio di ostruzione completa della laringe o della trachea e può provocare enfisema o addirittura il decesso [10]. Bilder et al. [10] hanno realizzato una revisione in tema di inalazione e ingestione di apparecchi ortodontici, rilevando che nella maggior parte dei casi (67\%) si trattava dellingestione di oggetti, con frequenza massima di episodi tra le donne. Allo stesso modo, il $95 \%$ di questi eventi awersi risultava essersi verificato al di fuori dello studio odontoiatrico e molto frequentemente riguardava un trattamento mascellare e trattamenti con apparecchi fissi. La maggior parte dei componenti ortodontici ha piccole dimensioni e, quando bagnati dalla saliva, la loro manipolazione diventa più difficile, con aumento del rischio di inalazione accidentale o ingestione [10].

L'inalazione accidentale e l'ingestione di apparecchi ortodontici è un fenomeno raramente riportato in letteratura. L'abituale posizione in decubito supino per migliorare l'accesso alla cavità orale aumenta il rischio di passaggio di strumenti nell'orofaringe. Tuttavia questi eventi awersi possono verificarsi anche quando il paziente è seduto durante il trattamento nello studio odontoiatrico. Sono comunque descritte alcune strategie per ridurre la loro incidenza durante la terapia [10].

\section{> Ipersensibilità al nichel e ad altri materiali ortodontici}

Riguardo all'allergia al nichel, gli studi suggeriscono un'elevata prevalenza del fenomeno nelle donne, a causa della prolungata esposizione all'allergene [12]. L'ipersensibilità a questo metallo non rappresenta un grave rischio per la salute del paziente. Tut- tavia l'ortodontista dovrebbe essere consapevole del problema e della possibilità che alcuni suoi pazienti possano soffrime [11]. I dati epidemiologici indicano che tra il $4,5 \%$ e il $28,5 \%$ della popolazione ha unipersensibilità al nichel, con una frequenza 10 volte maggiore tra le donne rispetto agli uomini. In ortodonzia, gli apparecchi in acciaio inox utilizzati contengono il $18 \%$ di cromo e l' $8 \%$ di nichel. Tuttavia, gli archi in nichel-titanio sono costituiti per più del $70 \%$ da nichel. L'evidenza di pazienti che sviluppano ipersensibilità a questo metallo a seguito dell'utilizzo di apparecchi ortodontici non è frequente in letteratura [11].

Alcuni fattori che possono portare a un aumento della sensibilità sono lírritazione meccanica, la lesione della pelle, un aumento di temperatura o salivazione e la durata di esposizione alla fonte di nichel. Si è concluso che una maggiore concentrazione di questo metallo, da 5 a 12 volte superiore a quella che sarebbe sufficiente a livello cutaneo, dovrebbe essere applicata alla mucosa orale per provocare una reazione di carattere dermatitico. Le proprietà ioniche, termiche, microbiologiche ed enzimatiche del cavo orale e l'esposizione a lungo termine agli apparecchi ortodontici durante il trattamento sono condizioni favorenti lo sviluppo di un'ipersensibilità al nichel [11].

Todd et al. [12] hanno studiato in 294 pazienti il rapporto tra lo sviluppo di un'allergia al nichel e un precedente contatto con questo metallo sotto forma di monili o apparecchi ortodontici. I ricercatori hanno rilevato che il $31,2 \%$ dei pazienti che avevano già avuto contatto con il metallo risultava allergico rispetto al $6,4 \%$ che non aveva avuto un pregresso contatto. Dai risultati di questo studio si può concludere che il trattamento ortodontico potrebbe ridurre la frequenza di allergia al nichel del 25-36\% e viene rafforzato il concetto che il contatto con allergeni orali possa portare a tolleranza immunologica.

Counts et al. [11] hanno riportato un caso di allergia al nichel associata a una barra transpalatale nella prima fase del trattamento ortodontico. L'apparecchio è stato rimosso e il paziente è stato inviato al parodontologo per eseguire una gengivectomia.

Thavarajah et al. [28] hanno esaminato il report degli eventi awersi (Adverse Event Report, AER) associati agli allineatori sequenziali utilizzando il database MAUDE (Food and Drug Administration's Manufacturer and User Facility Device Experience). Nel 5 anni del periodo di studio, sono stati registrati dal database MAUDE 175 casi di eventi awersi a causa dell'uso di allineatori sequenziali. Di tutti gli eventi awversi, 32 casi $(18,29 \%)$ sono stati diagnosticati come reazioni allergiche o ritenuti sospetti di esserlo, $20(11,43 \%)$ dei quali con reazione anafilattica e 4 
$(2,29 \%)$ con angioedema. Sono state più comunemente riportate lesioni coinvolgenti lingua, gola e labbra quali dolore, infiammazione e orticaria. Inoltre sono stati identificati 12 casi $(6,86 \%)$ che hanno riferito nausea, 11 (6,29\%) problemi gastrointestinali (disturbi gastrici, diarrea e vomito), 13 (7,43\%) problemi neuromuscolari (crampi muscolari, spasmi e dolore), $13(7,43 \%)$ tosse, $10(5,71 \%)$ cefalea persistente, $3(1,71 \%)$ febbre e $12(6,86 \%)$ problemi correlati al cuore [28].

Le manifestazioni di un'allergia a questo metallo possono includere dermatite nelle aree di contatto e stomatite. Le lesioni caratteristiche possono variare da un'eruzione o infiammazione all'eritema minore tendente al colore rosso, con o senza edema. I sintomi possono includere perdita del senso del gusto, ipersensibilità, dolore, eczema, prurito, cheilite angolare e sensazione di bruciore [11,12]. In generale la diagnosi si basa sull'anarnnesi del paziente, sui dati clinici e sui risultati dei test allergologici [11].

\section{> Ipersensibilità al paracetamolo \\ McAlinden et al. [22] hanno pubblicato un caso di ipersensibill- tà al paracetamolo in un paziente pediatrico di sesso maschile, in uno studio clinico randomizzato e controllato disegnato per confrontare ibuprofene e paracetamolo (acetaminofene) nel controllo del dolore ortodontico.}

\section{> Disfunzione cranio-mandibolare}

Nel 1990 Machen [29] ha riportato un caso di odontoiatria legale con presenza di dolore miofasciale e segni e sintomi di disfunzione cranio-mandibolare.

Alcuni autori, tra cui McNamara et al. [33], negano il rapporto fra trattamento ortodontico e segni e sintomi di disfunzione cranio-mandibolare; altri autori, come Michelotti et al. [34], non escludono invece tale correlazione.

\section{= 4.2 Effetti avversi associati ad apparecchi ortopedici intraorali}

Nel 2010 Fricton et al. [21] hanno stabilito, in una metanalisi, che apparecchi ortopedici intraorali usati nel trattamento dei disturbi temporo-mandibolari potrebbero determinare potenziali effetti awersi. Per esempio, diversi studi hanno descritto lo sviluppo di ulcere del tessuto mucoso, mal di denti, difficoltà di eloquio, mobilità dentale, alitosi, carie e modificazioni occlusali. Nello stesso anno Thiruvenkatachari et al. [20] hanno condot to uno studio clinico controllato randomizzato per confrontare l'apparecchio twin-block con dynamax nel trattamento della malocclusione di Classe II prima divisione. I risultati hanno dimostrato che la prevalenza di effetti awersi era maggiore nei pazienti trattati con dynamax (82\%) rispetto al twin-block (16\%) e riguardavano principalmente rotture dell'apparecchio e traumi della mucosa linguale e della lingua.

\section{$>$ Limitazione del movimento dentale}

Nei pazienti sottoposti a trattamento con bisfosfonati occorre prestare attenzione, quando si fa uso della laserterapia o di microviti per l'ancoraggio, ai traumi alla mucosa causati da retainer, alla chirurgia ortognatica e alle estrazioni per motivi ortodontici [31]. Nel 2011 Borromeo et al. [31] hanno esaminato le implicazioni cliniche dei bisfosfonati in odontolatria, individuando quattro casi nei quali questi farmaci hanno causato limitazioni del movimento dentale, anziché osteonecrosi mascellare. In ortodonzia i bisfosfonati trovano impiego nel trattamento dell'osteogenesi imperfetta nei bambini. Non è stato riscontrato alcun caso di osteonecrosi e le estrazioni dentali non risultano controindicate in questi pazienti. Sembra che l'età rappresenti un fattore protettivo per tali lesioni, sebbene non vi siano prove in tal senso [31].

\section{> Fallimento nella cementazione di tubi e bande}

Millett et al. [32], in una revisione sistematica, hanno osservato un tasso più elevato di fallimento della cementazione con i tubi molari rispetto alle bande; il dato era statisticamente significativo.

\section{> Decalcificazione}

Millett et al. [32] hanno parimenti determinato che vi era una maggiore decalcificazione nei molari sui quali erano state applicate bande in confronto ai tubi.

Le differenze nel successo della cementazione sono direttamente correlate con fattori quali la validità della corrispondenza tra le superfici, il tipo di adesivo utilizzato, l'esperienza dell'operatore, il paziente stesso e fattori meccanici. L'evidenza suggerisce che gli adesivi vetroionomerici siano più efficienti di quelli convenzionali nella prevenzione della decalcificazione [32]. In conclusione, da tutto quanto sopra esposto emerge límportanza di una corretta cartella clinica e dentale, cosi come di archivi diagnostici completi, del consenso informato e di un adeguato protocollo di trattamento [29].

\section{Conclusioni}

- Gli eventi awersi riportati in letteratura associati alla pratica ortodontica sono rappresentati da riassorbimenti radicolari,

142 | DeNTALCADMOS | 3/2017 
danno pulpare, danno parodontale, inalazione o ingestione di apparecchi ortodontici, rottura di tali apparecchi, allergia al nichel, ipersensibilità al paracetamolo, limitazione al movimento dentale e disfunzione cranio-mandibolare.

- La maggior parte degli eventi awersi assoriati alla pratica ortodontica ha gravità bassa o media.

- Al fine di trarre ulteriori conclusioni sono necessari trial clinici randomizzati controllati condotti con vari tipi di apparecchi ortodontici in pazienti con differenti classi di malocclusione.

- Sono necessarie più segnalazioni di eventi awersi non solo per produrre studi descrittivi ma anche per formulare raccomandazioni pratiche.

\section{CONFLITTO DI INTERESSI}

Gli autori dichiarano di non avere alcun conflitto di interessi.

\section{FINANZIAMENTI ALLO STUDIO}

Gli autori dichiarano di non aver ricevuto finanziamenti per il presente studio.

\section{BIBLIOGRAFIA}

1. Leape LL, Lawthers AG, Brennan TA, Johnson WG. Preventing medical injury, QRB Qual Rev Bull 1993;19(5):144-9.

2. Leape LL, Berwick DM. Five years after To Err is Human: what have we learned? JAMA 2005:293(19):2384-90.

3. World Health Organization. Conceptual Framework for the Interna tional Classification for Patient Safety. Version 1.1. Final technical report, 2009. http://www.who.int/about/copyright/es/index.html [accessed on 20 December 2014].

4. Weiser TG, Haynes AB, Dziekan G, Berry WR, Lipsitz SR, Gawande AA; Safe Surgery Saves Lives Investigators and Study Group. Effect of a 19-item surgical safety cherklist during urgent operations in a glohal patient population. Ann Surg 2010;251(5):976-80.

5. Perea B. Seguridad del paciente y odontologia. Cient Dent 2010;8(1):9-15,

6. Reukers EA, Sanderink GC, Kuijpers-Jagtman AM, van't Hof MA. Radiographic evaluation of apical root resorption with 2 different types of edgewise appliances. Results of a randomized clinical trial. J Orofac Orthop 1998;59(2):100-9.

7. Janson G, Bombonatti R, Brandão AG, Henriques JF, de Freitas MR. Comparative radiographic evaluation of the alveolar bone crest after orthodontic treatment. Am J Orthod Dentofacial Orthop 2003;124(2):157. 64

8. Brezniak N, Wasserstein A. Orthodontically induced inflammatory root resorption. Part l: the basic science aspects. Angle Orthod 2002:72(2):175-9.

9. Moriarty JD. Mucogingival considerations for the orthodontic patient. Curr Opin Periodontol 1996;3:97-102.

10. Bilder L, Hazan-Molina H, Aizenbud D. Medical emergencies in a dental office: inhalation and ingestion of orthodontic objects. J Am Dent Assoc 2011,142(1):45-52.

11. Counts AL, Miller MA, Khakhria ML, Strange S. Nickel allergy associated with a transpalatal arch applicance. J Orofac Orthop 2002;63(6):509. 15.

12. Todd DJ, Burrows D. Nickel allergy in relationship to previous oral and cutaneous nickel contact. Ulster Med J 1989;58(2):168-71.

13. Vaquero P, Perea B, Labajo E, Santiago A, García F. Reabsorción ro dicular durante el tratamiento ortodóncico: causas y recomendaciones de actuación. Clent Dent 2011:8(1):61-70.
14. Abboud M, Stark $\mathrm{H}$, Koeck B. Integration of all-ceramic restorations in reconstructive prosthetic dentistry: a case report. Quintessence Int 2004:35(2):103-7.

15. Meikle MC. The tissue, cellular, and molecular regulation of orthodontic tooth movement: 100 years after Carl Sandstedt. Eur J Orthod 2006;28(3):221-40.

16. de Jongh A, Aartman IH, Brand N. Trauma-related phenomena in anxious dental patients. Community Dent Oral Epidemiol 2003:31(1):52-8.

17. Klages $\mathrm{U}$, Weber $\mathrm{AG}$, Wehrbein $\mathrm{H}$. Approximal plaque and gingival sulcus bleeding in routine dental care patients: relations to life stress somatization and depression.J Clin Periodontol 2005,32(6).575-82.

18. da Fonseca MA. Adverse reaction to amoxicillin: a case report. Pediatr Dent 2000;22(5):401-4.

19. List T, Helkimo M. Adverse events of acupuncture and occlusal splint therapy in the treatment of craniomandibular disorders. Cranio 1992;10(4):318-24.

20. Thiruvenkatachari B, Sandler J, Murray A, Walsh T, O'Brien K. Comparison of Twin-block and Dynamax appliances for the treatment of Class II malocclusion in adolescents: a randomized controlled trial. Am J Orthod Dentofacial Orthop 2010:138(2):144.e1-9.

21. Fricton J, Look JO, Wright E, Alencar FG Jr, Chen H, Lang M, et al. Systematic review and meta-analysis of randomized controlled trials evalu. ating intraoral orthopedic appliances for temporomandibular disorders. J Orofac Pain 2010;24(3):237-54.

22. McAlinden RL, Ellis PE, Sandy JR. Report of an adverse incident in a randomized clinical trial. J Orthod 2005;32(3):203-5.

23. Brady J, Lewis $\mathrm{DH}$. Internal resorption complicating orthodontic tooth movement. Br J Orthod 1984;11(3):155-7.

24. Abuabara A. Biomechanical aspects of external root resorption in or thodontic therapy. Med Oral Patol Oral Cir Bucal 2007;12(8):E610-3.

25. Chan EK, Darendeliler MA. Exploring the third dimension in root re. sorption, Orthod Craniofac Res 2004;7(2):64-70.

26. de Castro LA, Vêncio EF, Mendonça EF. Epithelial inclusion cyst after free gingival graft: a case report. Int J Periodontics Restorative Dent 2007;27(5):465-9.

27. Mostafa YA, Iskander KG, El-Mangoury NH. latrogenic pulpal reactions to orthodontic extrusion. Am J Orthod Dentofacial Orthop 1991:99(1):30-4.

28. Thavarajah R, Thennukonda RA. Analysis of adverse events with use of orthodontic sequential aligners as reported in the manufacturer and user facility device experience datahase. Indian J Dent Res 2015;26(6);582-7.

29. Machen DE. Legal aspects of orthodontic practice: risk manage ment concepts. Excellent diagnostic informed consent practice and record keeping make a difference. Am J Orthod Dentofacial Orthop 1990:98(4):381-2.

30. Machen DE. Legal aspects of orthodontic practice: risk management concepts. Diagnosis, root resorption, and progress monitoring. Am J Orthod Dentofacial Orthop 1989:95(3):267-8.

31. Borromeo GL, Tsao CE, Darby IB, Ebeling PR. A review of the clinical implications of bisphosphonates in dentistry. Aust Dent j 2011:56(1):2-9.

32. Millett DT, Mandall NA, Mattick RC, Hickman J, Glenny AM. Adhe sives for bonded molar tubes during fixed brace treatment. Cochrane Datahase Syst Rev 2011;15(6):CDO08236.

33. McNamara JA Jr, Seligman DA, Okeson JP. Occlusion, orthodontic treatment, and temporomandibular disorders: a review. J Orofac Pain 1995;9(1):73-90.

34. Michelotti A, lodice $\mathrm{G}$. The role of orthodontics in temporomandibular disorders. J Oral Rehabil 2010;37(6):411-29. 